\title{
Nature, environnement et société en Allemagne
}

\section{Michel Deshaies}

\section{CpenEdition}

\section{Journals}

Édition électronique

URL : http://journals.openedition.org/rge/797

DOI : $10.4000 /$ rge. 797

ISSN : 2108-6478

\section{Éditeur}

Association des géographes de l'Est

\section{Édition imprimée}

Date de publication : 1 janvier 2007

Pagination : 9-14

ISSN : 0035-3213

\section{Référence électronique}

Michel Deshaies, "Nature, environnement et société en Allemagne », Revue Géographique de l'Est [En ligne], vol. 47 / 1 | 2007, mis en ligne le 22 décembre 2009, consulté le 22 septembre 2020. URL : http://journals.openedition.org/rge/797 ; DOI : https://doi.org/10.4000/rge.797

Ce document a été généré automatiquement le 22 septembre 2020.

Tous droits réservés 


\title{
Nature, environnement et société en Allemagne
}

\author{
Michel Deshaies
}

1 Une fois encore, la Revue Géographique de l'Est s'intéresse à l'Allemagne, mais sous un angle inédit, celui de la relation particulière qu'entretient la société allemande avec la nature et son influence sur la protection de l'environnement. Il s'agit ici plus précisément d'éclairer comment cette relation se traduit dans les représentations qu'on se fait de la nature, dans certains comportements sociaux et de montrer comment elle influence la politique de l'environnement et la vie économique et par là même, l'aménagement de l'espace.

2 La géographie, science de l'interface entre société et environnement, trouve là un objet d'étude particulièrement pertinent qui nécessite précisément des éclairages variés et complémentaires. L'étude géographique de l'environnement privilégie traditionnellement l'étude des relations d'interdépendance existant entre les sociétés et le cadre naturel ; ce qui fait qu'elle est souvent considérée comme un champ d'étude pour une géographie plutôt " physique ", analysant les relations entre milieux naturels et sociétés. Pour comprendre ces relations et surtout la façon dont elles s'inscrivent à la surface de la planète, il faut à la fois saisir le fonctionnement des milieux naturels et décrypter l'organisation économique et politique des sociétés, ainsi que les représentations que celles-ci se font de leur environnement. Les articles rassemblés dans ce numéro de la RGE montrent ainsi que le thème de l'environnement constitue aussi un champ d'étude fructueux pour des approches relevant plutôt de la géographie humaine, en l'occurrence culturelles, économiques et politiques.

3 Les géographes français et allemands qui ont participé à ce numéro ont des compétences et des orientations de recherche sensiblement différentes, qu'ils ont mobilisées pour analyser la relation complexe de la société allemande avec la nature et 
sa traduction dans l'espace géographique. On trouvera donc dans ces articles, des courants différents de la géographie :

- une géographie des représentations car, comme le montre Tobias Chilla, la nature n'est pas un objet neutre, mais une construction dans un certain contexte historique et social qui va influer sur la politique de protection de la nature et donc sur l'aménagement du territoire ;

- une géographie culturelle que Emmanuel Jaurand mobilise pour souligner que la représentation particulière que l'on se fait de la nature en Allemagne se traduit par des comportements sociaux originaux, comme le naturisme dont l'importance a aussi des répercussions sur la géographie du tourisme ;

- une géographie économique pour laquelle Christian Schulz montre que l'environnement est devenu récemment un thème d'étude, parce que précisément la protection de l'environnement est un secteur économique en plein essor, dans lequel l'Allemagne occupe une place dominante ;

- une géographie des transports et de l'aménagement urbain où Anne Hecker nous fait découvrir que, pour des raisons environnementales, l'Allemagne est devenu un champ d'expérimentation et d'innovation, où l'on cherche à inventer la ville « durable » du futur ;

- une géographie politique de l'exploitation des ressources, en l'occurrence énergétiques (Michel Deshaies), car en Allemagne tout particulièrement, ce problème est devenu une question centrale du débat sur l'environnement. L'Allemagne est en effet le théâtre de conflits environnementaux, liés à l'exploitation des ressources naturelles, qui opposent notamment les ONG écologistes aux sociétés de production d'électricité exploitant le lignite, le charbon ou l'énergie nucléaire.

4 À toutes ces questions géographiques, ces articles ont surtout pour ambition d'apporter des éléments de réflexion qui certes, montrent l'originalité de l'Allemagne, mais qui ce faisant, éclairent le débat actuel sur les relations entre société et environnement, notamment dans leurs dimensions culturelles, économiques et politiques. L'objectif est ainsi de montrer que l'étude des relations complexes existant entre les sociétés et l'environnement constitue un champ de recherche plein d'avenir pour la géographie et plus précisément pour une Géo-environnement (Veyret 1999), déjà très développée en Allemagne (Gebhardt, Glaser, Radtke, Reuber, 2007 ; Glaser, Gebhardt, Schenk, 2007).

\section{I. - Le besoin d'une nature idéalisée, à protéger}

5 Au visiteur qui sort des sentiers battus, l'Allemagne peut apparaître comme un pays étrange. Cette impression vient en partie de la relation particulière que les Allemands entretiennent avec la «nature» et de la façon dont ils modèlent leur cadre de vie. L'Allemagne offre en effet l'image très contrastée d'un pays densément peuplé, où la nature véritable est rare car le plus souvent très profondément transformée, mais où l'on déploie des efforts particulièrement importants pour préserver les espaces et les paysages considérés comme intéressants. Ceux-ci sont le plus souvent des paysages humanisés, les Kulturlandschaften, mais les premiers espaces protégés, dès le XIX siècle, ont été des sites correspondant à l'image que le romantisme se faisait de la nature (Tobias Chilla): rochers aux formes étranges comme le Drachenfels, le Teufelsmauer (photo 1), ou le Teutoburger Wald, évoquant les hauts faits des héros des légendes germaniques, forêts ou landes semblant constituer les derniers témoins du cadre de vie des anciens Germains. 
Malgré la diffusion de l'industrialisation et de l'urbanisation sur des surfaces croissantes (environ $13 \%$ du territoire en 2004), l'Allemagne a pu multiplier les zones naturelles protégées (on recense actuellement 6202 Naturschutzgebiete couvrant une superficie totale de $11320 \mathrm{~km} 2$, soit 2,6\% du territoire) qui voisinent et parfois même s'insèrent jusqu'au cœur des espaces les plus profondément bouleversés par les activités industrielles et minières d'une ampleur inégalée en Europe (Deshaies 2004, 2005). C'est ainsi que la liberté laissée à la puissante industrie extractive d'exploiter les ressources minérales dans d'immenses exploitations minières à ciel ouvert, semble en quelque sorte compensée par la multiplication des zones protégées. Même d'anciennes zones minières réhabilitées acquièrent le statut de zone naturelle et depuis la réunification allemande, on s'est aussi employé à protéger les espaces situés dans le no man's land qui s'étirait le long de l'ancien rideau de fer, ou encore dans des champs de manœuvre abandonnés par l'armée rouge.

Photo 1 : Le Teufelsmauer, près de Neinstedt (Saxe-Anhalt) est un des tout premiers « Naturschutzgebiet», créé dès 1852 (photo M.Deshaies, 2005).

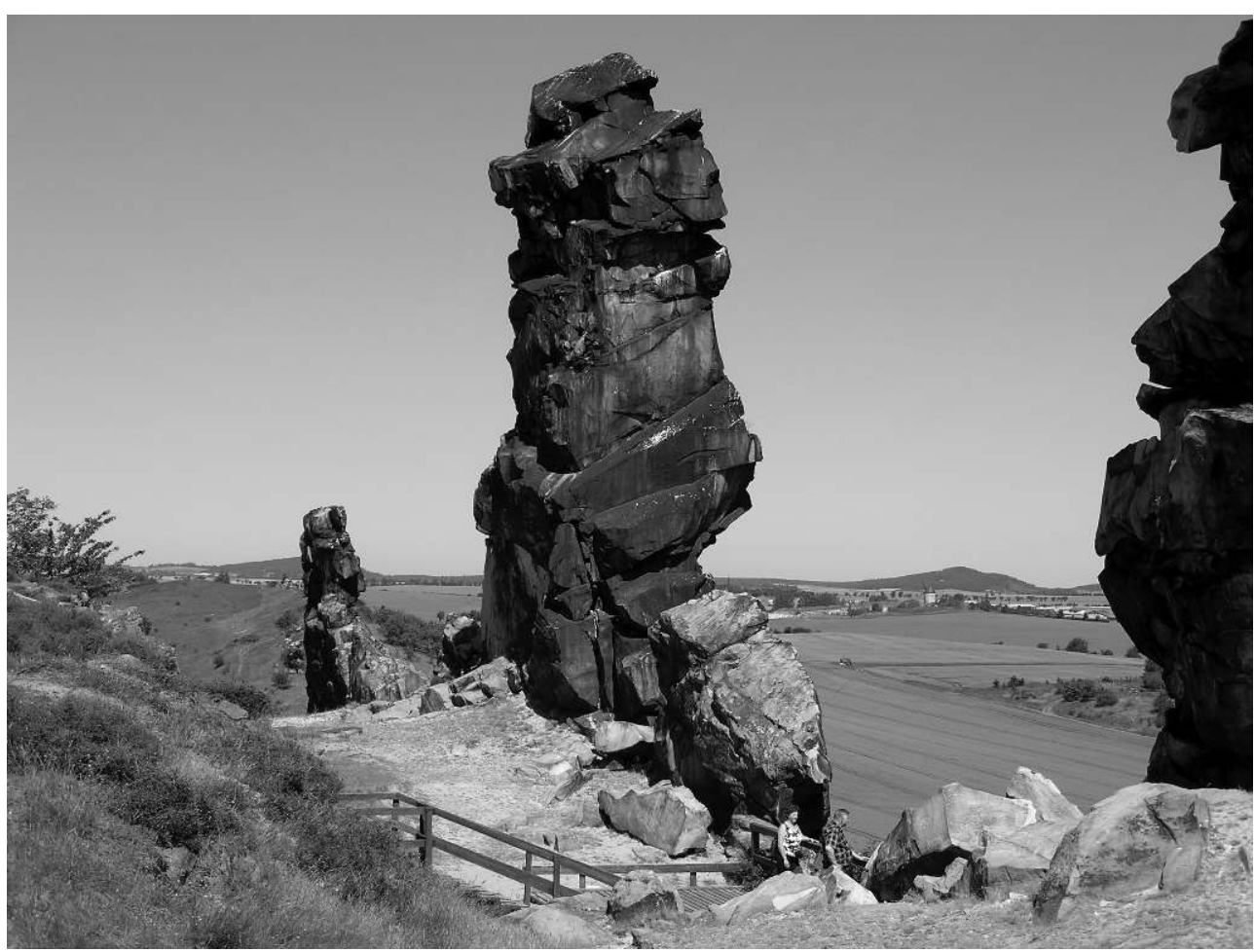

Les espaces relativement éloignés des grandes villes bénéficient d'un statut de protection plus ou moins strict qui concerne de vastes surfaces du territoire (figure 1). Les parties les plus strictement protégées, dans le cadre de l'un des 15 parcs nationaux, ou dans les réserves de biosphère, représentent des surfaces relativement limitées où les implantations humaines sont très faibles. C'est pourquoi ils semblent représenter l'image de la nature intacte. En fait, c'est rarement le cas, puisqu'il s'agit le plus souvent, à l'image de la lande de Lüneburg, le premier Naturschutzpark allemand, de paysages résultant de la dégradation de la végétation naturelle par d'anciennes formes d'exploitation agricole. L'essentiel des surfaces protégées, notamment une grande partie des massifs de moyenne montagne (le Mittelgebirge), l'est en fait de façon très relative, dans le cadre des parcs naturels (Naturparks) qui couvrent plus de $21 \%$ de la 
superficie du territoire. L'idée de base est de préserver une certaine qualité paysagère qui doit servir de support à un développement des activités touristiques et de loisirs.

Dans un pays très urbanisé, où l'espace est compté et de ce fait soigneusement aménagé, toutes ces zones «naturelles » ont vocation à servir d'espaces de détente de proximité (Naherholungsgebiet), destinés à compenser les contraintes de la vie dans les grandes agglomérations. En effet, l'urbanisation brutale et puissante du pays dans la deuxième moitié du XIX siècle n'a pas effacé et a peut-être même renforcé l'attachement de beaucoup d'Allemands à la nature. Le besoin de conserver un contact avec une nature de plus en plus menacée et restreinte par les agressions de la société urbaine et industrielle (Gebhardt et al. 1995) se traduit par exemple, par une pratique plus importante qu'en France des activités de nature telles que la randonnée pédestre ou à vélo, ou même par le caractère relativement banal du naturisme qui se définit luimême comme un « mode de vie en harmonie avec la nature » (Emmanuel Jaurand).

\section{II. - Un pays fortement engagé dans la défense de l'environnement}

9 Ce besoin de nature explique aussi que la société allemande accorde une place essentielle à la défense de l'environnement, comme en témoigne une législation très pointilleuse, inspirée par l'influence importante du lobby écologiste. En effet, celui-ci a trouvé en Allemagne de l'Ouest, à partir du début des années soixante-dix, un terrain particulièrement favorable. La formidable croissance économique des années cinquante et soixante a aussi provoqué une forte dégradation de l'environnement, avec notamment une inquiétante pollution de l'air et des cours d'eau traversant les grandes régions industrielles, en premier lieu la Ruhr. Le «Kohlenpott» (pot de charbon) semblait alors concentrer toutes les conséquences négatives de l'essor industriel. En réaction contre un problème dont une part de plus en plus importante de la population prend alors conscience, le gouvernement fédéral s'est fortement engagé et a mis en place, dès le début des années soixante-dix, de nombreuses lois visant à réduire les rejets de polluants dans l'air (Bundesemissionsschutzgesetz) et dans l'eau et un service de l'environnement (Umweltbundesamt) est créé en 1974.

Toutes ces mesures ont porté leurs fruits et contribué à améliorer nettement la qualité de l'environnement dans les grandes régions urbaines, à tel point que le vœu exprimé dans les années soixante " d'un ciel qui doit redevenir bleu au-dessus de la Ruhr » est maintenant réalisé. Il est vrai que dans ce cas de figure, c'est aussi l'effondrement de la production de charbon et la restructuration industrielle qui ont le plus contribué à réduire les sources de pollution. Cette politique ne se cantonne toutefois pas à la lutte contre les nuisances environnementales dans les régions urbaines. Elle se donne aussi pour ambition de préserver certains espaces, dans le cadre de la loi sur la protection de la nature de 1976 (Bundesnaturschutzgesetz). En effet, celle-ci a pour objectifs la préservation de la biodiversité, la protection des biotopes et de la qualité de l'air, des sols et des eaux, ainsi que la préservation de la qualité esthétique des paysages.

11 Néanmoins, beaucoup de citoyens allemands ne se sont pas contentés des mesures prises par l'Etat et se sont en conséquence engagés dans des « Bürgerinitiativen » qui, à partir du milieu des années soixante-dix, contestent de façon souvent radicale la société industrielle et se mobilisent contre les grands projets destructeurs de la «nature » (par exemple de construction d'autoroutes ou d'extension d'aéroports). C'est 
cependant surtout le développement de l'énergie électronucléaire qui va le plus mobiliser ces mouvements organisés par de puissantes ONG (BUND, Green Peace, NABU, WWF Deutschland). Les chantiers de construction des nouvelles centrales nucléaires, comme celui du surgénérateur de Kalkar en Rhénanie, deviennent alors, presque au sens propre, les champs de bataille de ces mouvements environnementalistes que l'accident de Tchernobyl en 1986, vient conforter dans leur attitude radicale. En Allemagne de l'Est, où la pollution fantastique générée par l'exploitation du lignite et une industrie chimique surdimensionnée atteignait pratiquement des records mondiaux, des mouvements de citoyens pour la protection de l'environnement se sont aussi constitués dans les années quatre-vingt pour investir un champ d'action politique très étroit. Néanmoins, la contestation ouverte de la dictature communiste en 1989 s'est aussi appuyé sur le terrain de l'environnement.

Depuis la réunification, qui a permis de prendre toute la mesure du saccage environnemental perpétré en ex-RDA par quarante ans d'économie dirigée, l'Allemagne a dû consacrer des moyens financiers gigantesques pour assainir et réhabiliter les héritages (Altlasten) menaçants pour l'environnement: la seule réhabilitation des immenses et sinistres friches minières des bassins de lignite et l'effacement des dangereux héritages de l'extraction de l'uranium ont nécessité, entre 1991 et 2005, plus de 12 milliards d'd'investissement (Zepp 2007), et la tâche est encore loin d'être achevée!

Figure 1 : Les grands ensembles d'espaces protégés en Allemagne.

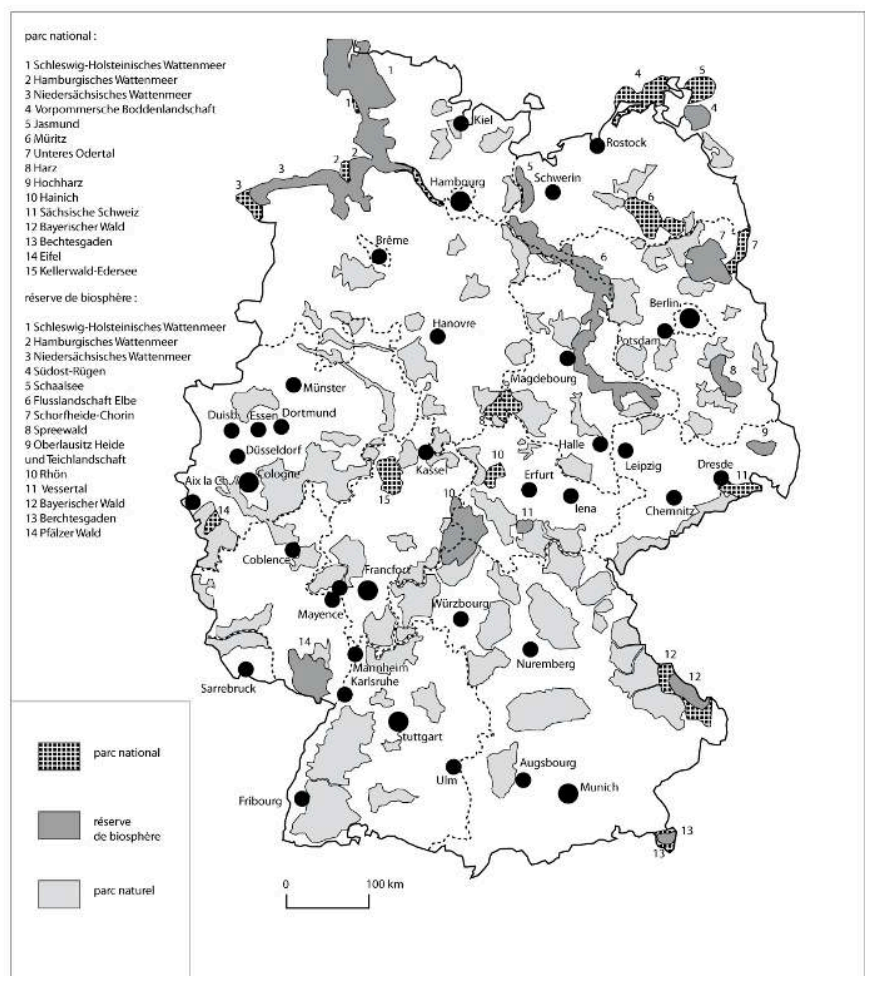

13 Cependant, si les besoins d'assainissement de l'environnement pèsent lourdement sur le budget de l'Etat, ils ont aussi généré le développement de tout un secteur économique spécialisé dans les techniques de dépollution et de plus en plus, dans les services de management environnemental. Mais au-delà, l'importance croissante 
accordée à la protection de l'environnement a soutenu le développement de nouvelles techniques de production industrielle ou d'énergie et plus largement, de nouveaux produits de consommation générant moins de pollution. Fortement encouragée par l'Etat, c'est donc une nouvelle industrie de l'environnement qui est en plein essor en Allemagne (Christian Schulz). En quelques années, celle-ci est devenue un secteur économique important dont les productions sont exportées en proportions croissantes. L'une des branches les plus dynamiques est celle des systèmes de production énergétique, qui est même en train de transformer l'image des paysages allemands, où la présence des éoliennes et des toits recouverts de cellules photovoltaïques est désormais banale (Michel Deshaies). Ainsi semble se réaliser la réconciliation de l'économie et de l'écologie que l'on tenait encore récemment pour inconciliables. Dans ce domaine des énergies renouvelables, l'Allemagne se veut exemplaire et a incontestablement pris beaucoup d'avance sur ses concurrents. Comme l'a montré le sommet européen de mars 2007 consacré à l'énergie, l'Allemagne veut jouer un rôle pilote et cherche à inventer le système énergétique du futur.

Cet avenir, elle le rêve sans énergie atomique, puisque qu'aux termes de la loi mise en application depuis 2002, toutes les centrales nucléaires allemandes doivent progressivement être fermées à l'horizon 2020. Pour réaliser cet idéal, il reste cependant à trouver le moyen de réduire considérablement la consommation énergétique et à développer beaucoup plus encore les énergies renouvelables (Michel Deshaies). C'est pourquoi, en Allemagne plus qu'ailleurs peut-être, on rêve d'une société où les comportements vertueux (pour l'environnement) se seront généralisés et, servis par des techniques nouvelles, permettront de réduire au minimum les besoins en énergie et plus largement en ressources prélevées sur la nature.

\section{III.- La conscience environnementale : entre utopie et réalité}

15 Cet idéal suppose le développement d'une conscience environnementale (Umweltbewusstsein), suffisamment importante pour provoquer une modification profonde des modes de vie et de consommation. Il est vrai que cette prise de conscience existe depuis longtemps en Allemagne et ne s'exprime pas seulement sur le terrain politique, mais se traduit aussi plus largement et plus concrètement par une adaptation des comportements dans certains domaines de la vie quotidienne. La volonté de limiter son impact sur l'environnement et de contribuer ainsi à la préservation de la «nature » se pratique depuis longtemps par le tri sélectif des ordures ménagères, ou par l'utilisation fréquente (en tout cas plus fréquente qu'en France) de la bicyclette pour les déplacements en ville. Le développement de ces comportements respectueux de l'environnement a été largement facilité par de multiples actions menées par les autorités locales et notamment par le développement d'un réseau adapté de transports en commun et la réalisation d'aménagements comme les pistes cyclables, dont la plupart des villes allemandes possède un réseau très étendu qui se prolonge dans les campagnes environnantes (Anne Hecker).

16 Plus globalement, c'est la ville telle qu'elle s'est développée à l'âge industriel, puis à celui de la banalisation de l'automobile, que l'on cherche à adapter et même à repenser, en réorganisant les systèmes de transport et même les quartiers d'habitation. Dans certaines villes comme Fribourg en Brisgau, la «ville durable » est déjà en gestation 
(Anne Hecker). De nouveaux quartiers sans voitures, constitués de maisons à basse consommation énergétique et utilisant principalement les énergies renouvelables (solaire, biomasse) ont été récemment réalisés. C'est un nouveau modèle de ville que l'on souhaite développer. Ce modèle retient d'autant plus l'attention que depuis peu, l'impact climatique de l'utilisation des énergies fossiles s'est imposé dans le débat sur la protection de l'environnement, en Allemagne comme ailleurs.

Pourtant, si ce que l'on pourrait appeler le modèle allemand de préservation de l'environnement, tel qu'il s'est développé depuis les années soixante-dix, a pu enregistrer de beaux succès, il est peut-être en train de trouver ses limites. En effet, tous les efforts qui ont été déployés jusqu'ici ont permis d'améliorer de manière significative l'environnement local, en réduisant la pollution de l'eau et de l'air dans les grandes régions urbaines et en préservant, ou même en restituant une qualité paysagère aux friches industrielles et minières, ainsi qu'à certains espaces plus ou moins éloignés des villes et destinés à servir de réservoirs de nature à leurs habitants. Le problème est que la question de la protection de l'environnement se pose de plus en plus à l'échelle globale et qu'à cette échelle, son efficacité se mesure à l'aune des volumes de gaz à effet de serre rejetés dans l'atmosphère par les activités humaines. Or, si les efforts consentis pour l'amélioration de l'environnement local (notamment depuis 1990 dans les nouveaux Länder) ont permis des progrès significatifs dans ce domaine, l'Allemagne reste aujourd'hui l'un des plus gros émetteur de $\mathrm{CO} 2$ par habitant de l'Union européenne (Michel Deshaies).

18 Cette situation est essentiellement due à l'importance qu'y conservent le charbon et le lignite pour la production d'électricité et donc aux choix énergétiques qui ont été faits et en partie justifiés pour des raisons environnementales. Le choix de sortir du nucléaire conforte en effet pour longtemps la part du charbon et du lignite dans le bilan énergétique de l'Allemagne. De manière plus anecdotique, elle est aussi due à certaines habitudes qui semblent aujourd'hui en contradiction avec la volonté de réduire l'impact sur l'environnement du mode de vie des Allemands, en l'occurrence la forte mobilité dans des voitures puissantes, roulant sans limitation de vitesse sur les autoroutes, ou les fréquents séjours dans des destinations lointaines. Cependant, changer ces habitudes pourrait se révéler être en contradiction avec l'économie, puisque ces mêmes voitures puissantes représentent à la fois la vitrine et une part importante des exportations du pays, tandis que les agences de voyage allemandes comptent parmi les entreprises de ce secteur les plus importantes au monde. Pourtant, à l'heure du réchauffement climatique, ce mode de vie énergivore paraitt de plus en plus incompatible avec la volonté affichée de réduire «l'empreinte écologique » de chaque citoyen. Celle-ci, qui peut se mesurer à la surface de forêt nécessaire pour absorber le dioxyde de carbone produit par la consommation énergétique, fait apparaître qu'avec leur mode de vie actuel, les Allemands devraient en théorie disposer d'une superficie en forêt équivalente à sept fois l'Allemagne.

Des hommes politiques du SPD et des Verts, ainsi que des scientifiques ont récemment dénoncé cette contradiction en recommandant aux citoyens désireux de contribuer à la réduction des émissions de gaz à effet de serre, de renoncer aux voyages en avion vers des destinations lointaines et de redécouvrir le charme des vacances en Allemagne, beaucoup moins consommatrices d'énergie : «Sylt statt Seychelles »! C'est ainsi que le président du Umweltbundesamt a fait observer qu'un voyage en avion vers le Sud-est asiatique produisait plus de six tonnes de dioxyde de carbone par personne, au lieu de 
$35 \mathrm{~kg}$ pour un voyage aller-retour en train de Berlin vers la mer Baltique. Ces prises de position assez radicales ont suscité un débat assez vif dont la presse s'est faite largement l'écho (voir en particulier les articles de die Zeit du 5 mars 2007 : « Sylt statt Seychelles", du Spiegel du 4 mars 2007: "Recht auf Reisen lässt sich niemand nehmen » et du 5 mars 2007 : «Deutschland-Reisen für den Klimaschutz », ainsi que de die Neue Epoche du 5 mars 2007: «Bundesbürger sollen für Klimaschutz Urlaub in Deutschland machen »), opposant notamment les détracteurs des voyages en avion au lobby des voyagistes. En effet, si ces recommandations devaient être suivies d'effets, cela pourrait avoir beaucoup de conséquences économiques sur l'industrie touristique mondiale, étant donné que les Allemands dépensent plus de 60 milliards d'par an dans leurs voyages à l'étranger.

Il est vrai que les agences de voyage et les compagnies aériennes ont déjà imaginé une réplique, en proposant de " compenser » les émissions de dioxyde de carbone générées par les voyages lointains en avion, par le financement de projets écologiques (par exemple, des actions de reboisement ou le développement des énergies renouvelables), de préférence dans des pays du Tiers-monde (articles du Spiegel du 3 novembre 2006 : «Zahlen für das Gewissen" et du 5 mars 2007: "Reiseverband fordert 3 LiterFlugzeug "). Au pays de Luther, cette façon d'acheter son bout de paradis tropical en versant une obole aux pauvres est interprétée par certains comme un nouveau trafic des indulgences. Il est clair en tout cas que, dans un pays qui est considéré comme un modèle dans le domaine de la protection de l'environnement (de la même façon que, dans les années soixante, la République fédérale était un modèle économique), la question de l'adaptation du mode de vie aux exigences environnementales peut difficilement être esquivée.

21 En effet, une enquête récente réalisée par le service fédéral de l'environnement (Umweltbundesamt) a montré que $66 \%$ des citoyens allemands considéraient que l'Allemagne devait jouer un rôle pilote au niveau mondial dans le domaine de la protection de l'environnement (www.umweltbewusstsein.de). Dans la même enquête, il apparaissait que $80 \%$ des Allemands se sentaient plus ou moins fortement menacés par les conséquences du changement climatique et jugeaient important ou très important de réduire les émissions de gaz à effet de serre. Mais pour y parvenir, les citoyens sont plus partagés sur les moyens. Si le développement des énergies renouvelables est plébiscité, seule une faible minorité de citoyens envisagent de pouvoir contribuer personnellement à la protection de l'environnement, en économisant l'énergie et notamment en renonçant aux vacances plus ou moins lointaines à l'étranger pratiquées par les trois quarts des touristes allemands.

Il est vrai qu'il y a souvent loin des intentions à la réalité. Si la consommation totale d'énergie par habitant a effectivement diminué depuis 1990, c'est essentiellement une conséquence de la restructuration économique de l'Est qui a mis un terme à la gabegie énergétique de l'économie dirigée. Par contre, si depuis 1993 la consommation d'énergie par habitant reste stable, on observe une nette augmentation de la consommation d'électricité qui a progressé de pratiquement $20 \%$ (Michel Deshaies). Dans ces conditions, toutes les perspectives visant, à brève échéance, à assurer l'approvisionnement énergétique futur du pays, en réduisant considérablement la consommation de chacun, paraissent pour le moins utopiques et supposent en tout cas un profond changement des modes de vie, difficilement envisageable dans le cadre de la société actuelle. 


\section{BIBLIOGRAPHIE}

DESHAIES M. (2004). - Energie et paysages en Allemagne : les enjeux environnementaux, Annales de Géographie, n 637, p. 27-51.

DESHAIES M. (2005). - Exploitation minière et paysages, thèse d'HDR, Université de Nancy 2, 481 p. GEBHARDT H. REUBER P., STEGMANN B.A. (1995). - Ortsbindung im Verdichtungsraum - Theoretische Grundlagen, methodische Ansätze und ausgewählte Ergebnisse, in : GEBHARDT H. \& SCHWEIZER G. (Hrsg) : Zuhause in der Großstadt. Ortsbindung und räumliche Identifikation im Verdichtungsraum. Kölner Geographische Arbeiten 61, p. 3-58.

GEBHARDT H., GLASER R., RADTKE U., REUBER P. (2007). - Geographie, Elsevier, Spektrum Akademischer Verlag, $1096 \mathrm{p}$.

GLASER R., GEBHARDT H., SCHENK W. (2007). - Geographie Deutschlands, Wissenschaftliche Buchgesellschaft, $280 \mathrm{p}$.

VEYRET Y. (1999). - Géo-environnement, Campus, SEDES, 160 p.

ZEPP H. (2007). - Hazardscapes - ausgewählte Problemräume, in : GLASER R., GEBHARDT H., SCHENK W. : Geographie Deutschlands, p. 216- 223.

\section{Publications en ligne : les articles cités ci-dessus sont consultables en ligne sur les sites suivants}

Zahlen für das Gewissen » et du 5 mars 2007, der Spiegel du 3 novembre 2006 : http://

www.spiegel.de/reise/

aktuell/0,1518,446389,00html

Sylt statt Seychelles, die Zeit, 5 mars 2007 : http://zeus.zeit. de/text/online/2007/10/

klimadebatte

Recht auf Reisen lässt sich niemand nehmen, der Spiegel du 4 mars 2007 : http://www.spiegel.

de/reise/aktuell/0,

1518,469943,00.html

Deutschland-Reisen für den Klimaschutz, der Spiegel du 4 mars 2007 : http://www.spiegel.de/ politik/deutschland/

$0,1518,469742,00 . h t m l$

Bundesbürger sollen für Klimaschutz Urlaub in Deutschland machen, Die Neue Epoche du 5 mars 2007 : http://www. dieneueepoche.com/articles/2007/03/04/94884p.html

Reiseverband fordert 3 Liter-Flugzeug, der Spiegel du 5 mars 2007 : http://www.spiegel.de/reise/ aktuell/0,1518,470004,

00.html 
AUTEUR

MICHEL DESHAIES

CERPA - Université de Nancy 2 - 23, Bd Albert Ier, 54015 Nancy CEDEX 